\title{
Microinvasion of liver metastases from colorectal cancer: predictive factors and application for determining clinical target volume
}

Yang Qian ${ }^{1}$, Zhao-Chong Zeng ${ }^{1 *}$, Yuan $\mathrm{Ji}^{2}$ and Yin-Ping Xiao ${ }^{2}$

\begin{abstract}
Objectives: This study evaluates the microscopic characteristics of liver metastases from colorectal cancer (LMCRC) invasion and provides a reference for expansion from gross tumor volume (GTV) to clinical targeting volume (CTV).

Methods: Data from 129 LMCRC patients treated by surgical resection at our hospital between January 2008 and September 2009 were collected for study. Tissue sections used for pathology and clinical data were reviewed. Patient information used for the study included gender, age, original tumor site, number of tumors, tumor size, levels of carcinoembryonic antigen (CEA) and carbohydrate antigen 199 (CA199), synchronous or metachronous liver metastases, and whether patients received chemotherapy. The distance of liver microinvasion from the tumor boundary was measured microscopically by two senior pathologists.
\end{abstract}

Results: Of 129 patients evaluated, 81 (62.8\%) presented microinvasion distances from the tumor boundary ranging between $1.0-7.0 \mathrm{~mm}$. A GTV-to-CTV expansion of 5, 6.7, or $7.0 \mathrm{~mm}$ was required to provide a 95, 99, or $100 \%$ probability, respectively, of obtaining clear resection margins by microscopic observation. The extent of invasion was not related to gender, age, synchronous or metachronous liver metastases, tumor size, CA199 level, or chemotherapy. The extent of invasion was related to original tumor site, CEA level, and number of tumors. A scoring system was established based on the latter three positive predictors. Using this system, an invasion distance less than $3 \mathrm{~mm}$ was measured in $93.4 \%$ of patients with a score of $\leq 1$ point, but in only $85.7 \%$ of patients with a score of $\leq 2$ points.

Conclusions: The extent of tumor invasion in our LMCRC patient cohort correlated with original tumor site, CEA level, and number of tumors. These positive predictors may potentially be used as a scoring system for determining GTV-to-CTV expansion.

Keywords: Liver metastases, Colorectal cancer, Radiotherapy, Pathological characteristics, Tumor invasion

\section{Summary}

Data from 129 patients with liver metastases from colorectal cancer (LMCRC) were used to analyze factors associated with hepatic metastatic microextensions. The extent of invasion was positively correlated with the original primary tumor site, CEA levels, and number of tumors. These predictors may potentially be used as a scoring system for determining gross tumor volume (GTV) to clinical tumor volume (CTV) expansion.

\footnotetext{
* Correspondence: zeng.zhaochong@zs-hospital.sh.cn

'Department of Radiation Oncology, Zhongshan Hospital, Fudan University, 136 Yi Xue Yuan Road, Shanghai 200032, China

Full list of author information is available at the end of the article
}

\section{Introduction}

Colorectal cancer is one of the most common gastrointestinal carcinomas, and worldwide incidence and mortality rates are continually increasing. The five-year survival rate after radical surgery is $40-56 \%$. The major failure pattern involves liver metastases. During followup observation, $50-70 \%$ of patients diagnosed with colorectal cancer exhibit liver involvement, which presents as a solitary nodule in one-half of these patients [1]. In approximately $25 \%$ of cases diagnosed with colorectal cancer, liver metastases are found at the time of diagnosis. Among those without liver metastases at the 
time of diagnosis, $80-90 \%$ will have liver metastases within 2-3 years after surgery [2].

In 2010, colorectal cancer guidelines proposed that carefully selected patients should be considered for conformal radiation therapy and included in some clinical trials. Studies in the literature show that stereotactic body radiation therapy (SBRT) for liver metastases from colorectal cancer (LMCRC) is an effective ablation therapy with a favorable toxicity profile $[3,4]$. Conformal radiotherapy accurately shapes the high-dose radiotherapy beam closely around the outline of the visible tumors and subclinical lesions. Precise outlining of the gross tumor volume (GTV) and the clinical targeting volume (CTV) can focus the radiation dose to the target tumor and reduce exposure and toxicity to normal tissue.

GTV is the volume of the visible tumor. CTV includes GTV and is defined as the volume of tissue that has a significant probability of containing microscopic tumor extensions (microextensions), which represents subclinical diseases. Few studies have analyzed microextension characteristics in liver metastasis. This knowledge deficit limits the application of conformal radiotherapy for LMCRC. The objectives of this study were to perform histological quantification of microextension at liver metastatic lesions, and to correlate pathological macroscopic tumor dimensions with MRI measurements to define CTV as precisely as possible.

\section{Methods}

\section{Ethics approval and patients in the study}

This study was approved by the ethical review board of Zhongshan Hospital, Fudan University and in compliance with the Helsinki Declaration of 1975, as revised in 2000. Between January 2008 and September 2009, 129 patients with liver metastasis from colorectal cancer underwent radical surgical resection at Zhongshan Hospital. Patient selection was based on the following criteria. Patients must have undergone a radical resection for liver lesions at our hospital, and must have been pathologically diagnosed with metastatic adenocarcinoma. To avoid potential influences on the presence of microextensions, patients may not have received recent local treatment for liver metastatic lesions. Complete clinical data must have been available for patients, including tumor information and laboratory values. Normal liver tissue surrounding tumors must have had at least a $1-\mathrm{cm}$ margin extending beyond the tumor boundary, so that all microextensions could be observed properly. Many lesions are excluded from radiotherapy due to possible radiation damage of normal liver [5, 6]. Patients were excluded from the current study if more than four lesions were detected by preoperative radiography or laparotomy. If more than one lesion was present, we selected the largest one to evaluate.

\section{Pathological analysis}

After radical resection, surgical specimens were analyzed by surgeons to determine the pathological boundary type and maximum diameter. Then, some specimens were fixed in phosphate-buffered saline (PBS) containing $10 \%$ $(\mathrm{v} / \mathrm{v})$ formalin; in this study, these are designated as pathology specimens. Unfixed specimens are designated as surgical specimens. All specimens were sectioned into $1.5 \times 1.0 \times 0.4-\mathrm{cm}$ slices, dehydrated, and then embedded in paraffin. Tissue blocks were prepared for routine histological examination, and $5-\mu \mathrm{m}$ sections on slides were stained with hematoxylin and eosin (H\&E) for light microscopy. Three hundred slides from these specimens were reviewed. To avoid variations between observations, a single pathologist assessed all specimens to identify microscopic evidence of microextension.

\section{Analysis and definitions of microextensions}

On each histological slide, tumor margins were assessed on the cut surface by gross findings and then outlined by marker pen with indelible ink. A microextension was defined as an extension of the tumor through the marked margin, and was analyzed by light microscopy using an Olympus BX 40 (Olympus; Tokyo, Japan) at low-power magnification $(\times 40)$ to identify the boundary between the tumor and normal liver tissues. These observations also were confirmed by examination at a higher magnification $(\times 100$ or $\times 400)$. On each slide, the maximum distance to normal liver tissue around the tumor periphery was determined using an eyepiece with micrometer. If two or more microinvasions were observed (Fig. 1f), the longest invasive distance was recorded. If the primary tumor was completely or partially surrounded by a fibrous pseudocapsule (Fig. 1b), the microextension was considered to be the maximum distance from the outer border of the pseudocapsule to the outer boundary of visible nests of tumor cells [7]. If a fibrous pseudocapsule surrounding the primary tumor was absent (Fig. 1c), the tumor mass was defined as the area that lacked (or essentially lacked) liver parenchyma interspersed between tumor cells as determined by microscopic examination. If the tumor had a capsule and did not exhibit microinvasion (Fig. 1d), the microextension was considered to be the maximum distance from the outer border of the pseudocapsule to the outer boundary of visible nests of tumor cells. The tumor border was defined as the line along whose length liver tissue and nests of tumor cells became interchanged; in this case, the microextension was defined as the distance from the primary tumor border to the outer boundary of visible nests of tumor cells (Fig. 1) [8].

Magnetic resonance imaging and computed tomography We collected data from magnetic resonance imaging (MRI) and computed tomography (CT) images to compare 


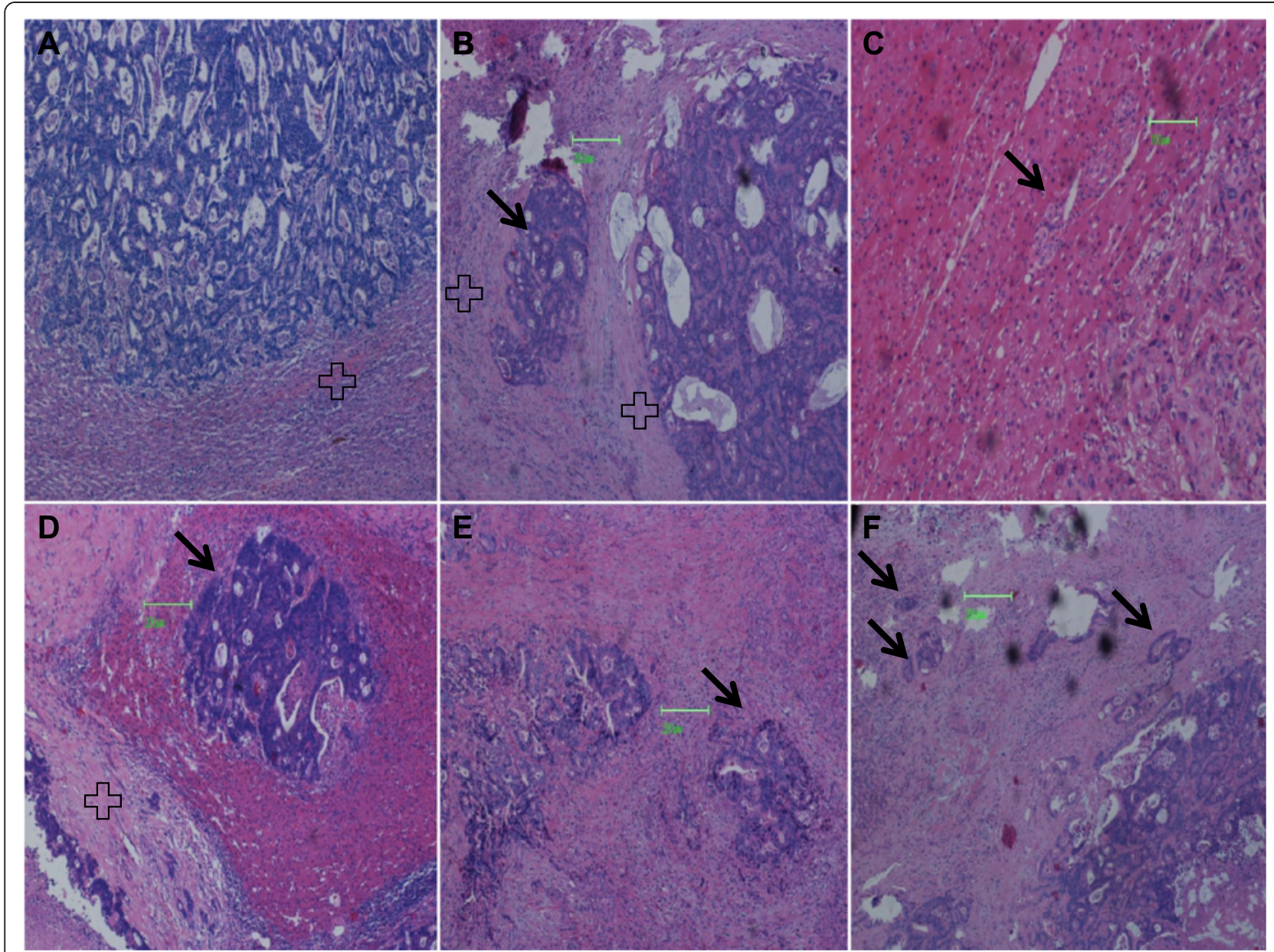

Fig. 1 Survey of observations of liver microinvasion from colorectal tumor metastases. a Tumor with a capsule (black cross) and showing a distinct boundary. b Tumor and microinvasions (black arrows) with capsules. c Tumor and microinvasions without capsules. d Tumor with a capsule that does not exhibit microinvasions. e Irregularly protruding microinvasions. f Multiple microinvasions (black arrows) with different sizes and shapes that show obscure boundaries

with the pathological measurements. To allow as little time as possible for additional tumor development before performing surgery, the scans had to be performed within 3 days before resection. Of the two imaging modalities, MRI was preferred to CT for its superior assessment of malignant focal liver lesions $[9,10]$.

Of the 129 patients in this study, only 49 underwent MRI at our hospital. An MRI scan of the liver was performed with a $1.5 \mathrm{~T}$ MR scanner (SignaHDxt; General Electric, WI, USA). This preoperative MRI included a T2-weighted (w) fast-spin echo (FSE) single-shot (SS) sequence, a T1-w gradient-echo (GE) sequence, and a T1-w dynamic multiphase gadolinium-enhanced (DMGE) sequence (Fig. 2a-d). All MRI sequences were performed while the patient held his breath (after exhalation). The T2-w FSE FS sequence also was performed with the system triggered to expiration. The slice thickness for T1-w GE and T2-w FSE SS sequences was $8 \mathrm{~mm}$, and for the T1-w DMGE sequence it was $5 \mathrm{~mm}$. Liver metastases vary in their T1 and T2 signal intensities but are usually prolonged, which results in hypointensity to isointensity on T1-w images and isointensity to hyperintensity on T2-w images. Some metastases may show a hyperintense halo of viable tumor surrounding a central region of hypointensity due to necrosis. The pathologically determined GTV correlated best with unenhanced MRI compared with venous enhanced MRI. The tumor contours revealed by T1-w GE were reviewed by an experienced liver radiologist.

\section{Comparison of preoperative MRI with macroscopic pathology}

To define the GTV by radiography, we calculated that the coefficient of specimen contraction during fixation for a pathology slide was $90.1 \%$, as reported by Bi et al. [11] from their analysis of 66 primary liver carcinoma specimens. The pathology specimens, which included both tumor and normal liver tissues, were cut into a regular rectangle of $2 \times 1.5 \mathrm{~cm}$. The width and length of 

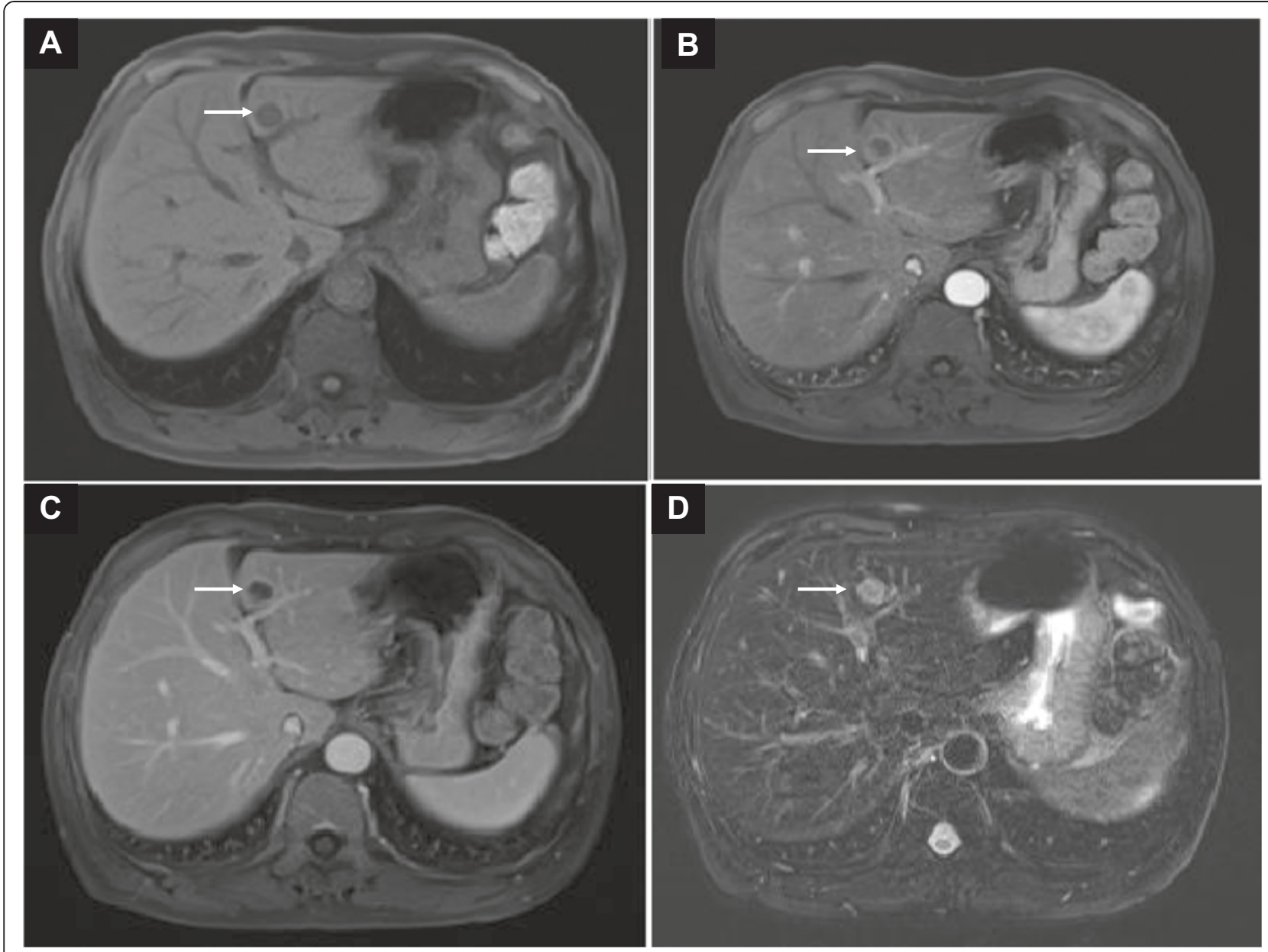

Fig. 2 Liver metastasis (white arrow) of preoperative magnetic resonance images. a T1-w GE. b T1-W arterial enhanced. c T1-w venous enhanced. d T2-W

the cut tissue were measured. Two to four pieces of the specimens were placed into an automatic tissue hydroextractor and subjected to a dehydration cycle. H\&Estained slides were prepared using routine methods, and the resulting tissue sections were measured. Finally, the specimen contraction coefficient was calculated based on the two measured specimen sizes.

\section{Definitions of clinical factors}

The preoperative evaluation included a medical history, physical examination, complete blood-cell count, liver function tests, measurements of carcinoembryonic antigen (CEA) and carbohydrate antigen 19-9 (CA199) levels, chest X-ray, abdominal ultrasonography, and enhanced CT or MRI (or both). Levels of CEA and CA199 tumor markers were measured using an electrochemiluminescence immunoassay system (Roche Modular E170). Liver functions were tested by an automated method and analyzed statistically (Hitachi 7600-120). All laboratory tests were performed less than 1 week before surgery at the Clinical Laboratory Department of Zhongshan Hospital. The presence of multiple lesions is defined as more than one lesion but less than four lesions. Chemotherapy is defined as having undergone at least one chemotherapeutic regimen before live surgery. Liver metastases are defined as synchronous when they occur within 6 months after the original tumor surgery and as metachronous when they occur after 6 months. The surgeons specified the original tumor site as rectum when it was located within $<12 \mathrm{~cm}$ from the perianal skin border, and as colon when it was located in cecum, ascending colon, transverse colon, descending colon, or sigmoid colon. All patients had hepatic lesions that were consistent with metastases from a histologically documented colorectal carcinoma.

\section{Statistical analysis of data}

Statistical analysis was performed using SPSS version 19.0 (SPSS Inc., Chicago, IL). The association of characteristics with microinvasion distance was analyzed by 
chi-square test for categorical variables, and by binary logistic regression for predictor variables of the significant parameters. $P$ values $\leq 0.05$ were considered significant.

\section{Results}

Demographic data

One hundred and twenty-nine LMCRC patients who fulfilled the specified criteria were recruited, including 75 men and 54 women. The patient ages ranged from 32 to 80 years, with a mean of 59 years.

Microextension was noted in $62.8 \%(81 / 129)$ of the patients. Microinvasion was assessed from H\&E-stained sections. Microscopic analysis of tissue specimens from these patients revealed two microinvasion patterns in LMCRC patients. In one, the region of microextension and tumor foci was separated by normal liver tissue (Fig. 1b). The second pattern was irregular protrusion extending beyond the tumor (Fig. 1e). The microextension distances ranged from 1-7 $\mathrm{mm}$, with an average distance of $1.50 \pm 1.51 \mathrm{~mm}$. The microextension distance was $1-2 \mathrm{~mm}$ in $48(37.2 \%)$ patients, $2.1-4.0 \mathrm{~mm}$ in 24 (18.6\%) patients, and $4.1-7.0 \mathrm{~mm}$ in $9(7 \%)$ patients. This distance had to be expanded to $5 \mathrm{~mm}$ during microscopy to include at least $98 \%$ of all microextensions.

\section{Factors associated with the presence of microextensions}

Clinical factors associated with the presence of microextension are listed in Table 1. The data indicated that the presence of microextensions was associated with high serum levels of CEA $(P=0.002)$, primary tumor site in colon $(P=0.008)$, and multiple lesions $(P=0.045)$.

\section{Scoring system to estimate risk of microextensions with} varying distances for all patients

Plots showing the cumulative distribution of microinvasion according to the clinicopathologic factors are presented in Fig. 3. We found that among patients with microinvasion distance of $\leq 2.4 \mathrm{~mm}, 94.3 \%$ had CEA levels of $\leq 5 \mathrm{U} / \mathrm{ml}$. When the microinvasion distance was $\leq 4.3 \mathrm{~mm}$, sensitivity was approximately $95 \%$ for patients in which the primary tumor site was rectum. A microinvasion distance of $\leq 4 \mathrm{~mm}$ was associated with a sensitivity of $95 \%$ for patients with a single liver metastatic lesion (unilesional). The best single predictor for microinvasion was the CEA level.

To improve the success of prediction for microinvasion, we developed a scoring system based on a combination of the associated factors that were identified. Patients were assigned a score of 0 for each of the following: CEA level $\leq 5$; original site of primary tumor in rectum; and unilesional liver metastasis. Otherwise, patients received a score of 1 for factors not meeting those criteria. To account for $\geq 95 \%$ probability of microinvasion, one must select an expansion of $2 \mathrm{~mm}$
Table 1 Factors associated with the presence of microextension

\begin{tabular}{|c|c|c|c|c|c|}
\hline \multirow{2}{*}{$\begin{array}{l}\text { Clinicopathological } \\
\text { factor }\end{array}$} & \multirow[t]{2}{*}{$n$} & \multicolumn{2}{|c|}{ Microextension, $n$ (\%) } & \multirow[t]{2}{*}{$x^{2}$} & \multirow[t]{2}{*}{$P$-value } \\
\hline & & Absent & Present & & \\
\hline Sex & & & & 0.790 & 0.598 \\
\hline Male & 75 & 28 (37.3\%) & 47 (62.7 \%) & & \\
\hline Female & 54 & 20 (37.0\%) & $34(63.0 \%)$ & & \\
\hline Age & & & & 0.794 & 0.624 \\
\hline$<65$ years & 93 & 34 (36.6 \%) & $59(63.4 \%)$ & & \\
\hline$\geq 65$ years & 36 & 14 (38.9\%) & $22(61.1 \%)$ & & \\
\hline $\begin{array}{l}\text { Synchronous or } \\
\text { metachronous }\end{array}$ & & & & 0.982 & 0.965 \\
\hline Synchronous & 70 & 25 (35.7\%) & $45(64.3 \%)$ & & \\
\hline Metachronous & 59 & $23(39 \%)$ & 36 (61 \%) & & \\
\hline Original site & & & & 3.185 & 0.009 \\
\hline Rectum & 48 & 25 (52.1\%) & $23(47.9 \%)$ & & \\
\hline Colon & 81 & $23(28.4 \%)$ & $58(71.6 \%)$ & & \\
\hline Number of tumors & & & & 2.537 & 0.045 \\
\hline 1 & 85 & 38 (44.7\%) & $47(55.3 \%)$ & & \\
\hline$>1$ & 44 & 10 (22.7 \%) & $34(77.3 \%)$ & & \\
\hline Max tumor diameter & & & & 1.345 & 0.52 \\
\hline$<3 \mathrm{~cm}$ & 39 & 17 (43.6 \%) & $22(56.4 \%)$ & & \\
\hline$\geq 3 \mathrm{~cm}$ & 90 & 31 (34.4\%) & $59(65.6 \%)$ & & \\
\hline CEA (ng/ml) & & & & 4.805 & 0.001 \\
\hline$\leq 5$ & 35 & 20 (57.1\%) & $15(42.9 \%)$ & & \\
\hline$>5$ & 94 & 28 (29.8\%) & 66 (70.2 \%) & & \\
\hline CA199 (U/ml) & & & & 0.478 & 0.114 \\
\hline$\leq 37$ & 68 & 24 (35.3\%) & $44(64.7 \%)$ & & \\
\hline$>37$ & 61 & 24 (39.3\%) & $37(60.7 \%)$ & & \\
\hline Chemotherapy & & & & 0.906 & 0.817 \\
\hline Yes & 55 & 21 (38.2 \%) & 34 (61.8\%) & & \\
\hline No & 74 & 27 (36.5 \%) & 47 (63.5 \%) & & \\
\hline
\end{tabular}

for a score of $\leq 1$, and an expansion of $5 \mathrm{~mm}$ for a score of $\geq 2$ (Fig. 3d).

\section{Tumor sizes determined from radiographs, surgical specimens, and pathology specimens}

In samples from the 49 patients undergoing a preoperative MRI scan at our hospital, only 49/129 tumors (38\%) received the same boundary classifications based on gross analyses of surgical specimens and radiographs. The mean tumor diameter measured $3.3 \mathrm{~cm}$ (range, 0.3$9.0 \mathrm{~cm}$ ) in radiographs, $3.5 \mathrm{~cm}$ (range, $0.8-9.5 \mathrm{~cm}$ ) in surgical specimens, and $3.2 \mathrm{~cm}$ (range, $0.8-9.5 \mathrm{~cm}$ ) in pathology specimens. Regression analysis of tumor size in radiographs, surgical specimens, and pathology specimens revealed that the three measurements were significantly correlated $(P<0.001)$. This was especially true of 


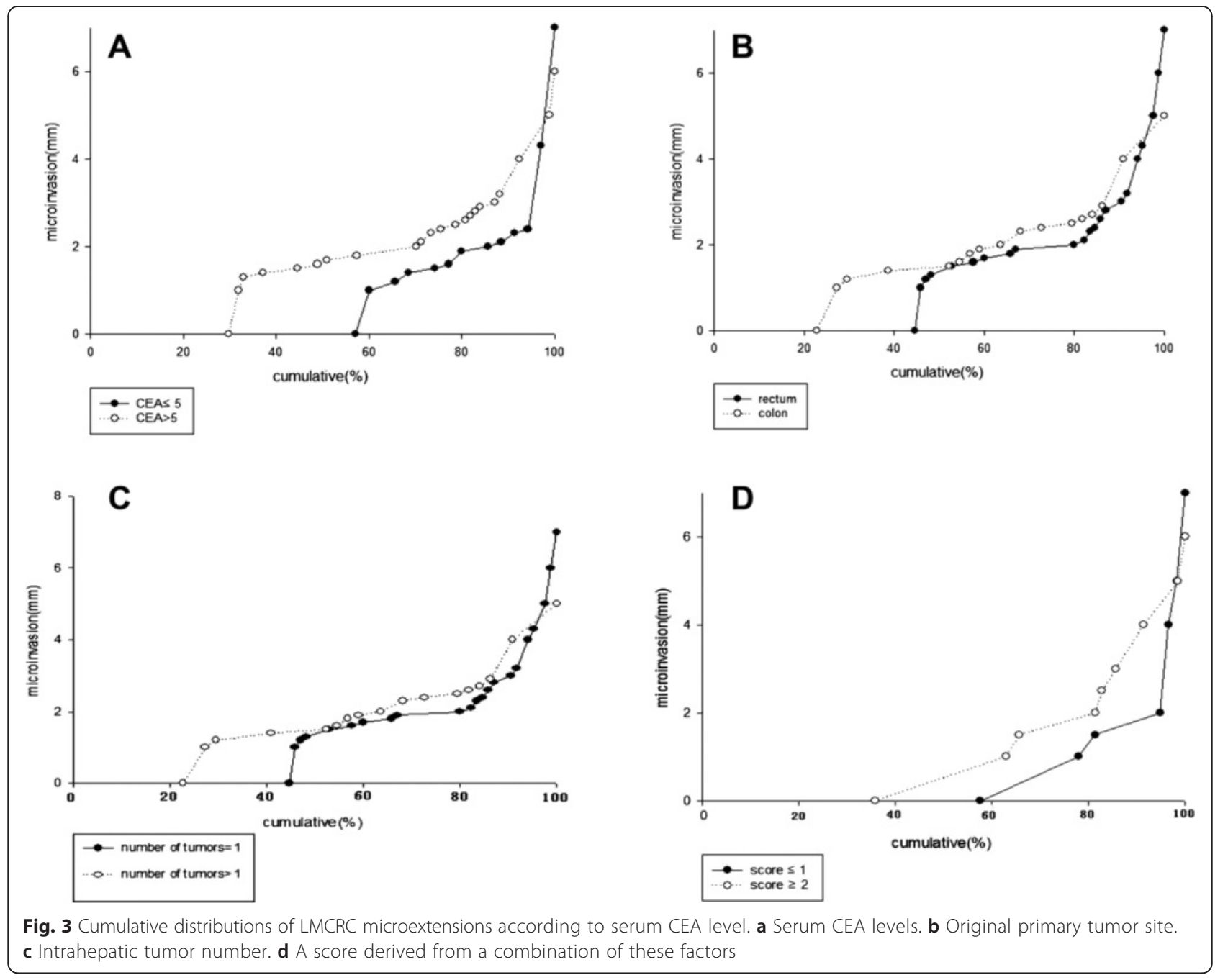

measurements taken from radiographs and surgical specimens. Measurements from pathology specimens were slightly smaller than those taken from radiographs $(r=0.92, P<0.001)$. A comparison of radiographic measurements with those obtained from pathology specimens showed that radiographic measurements were larger in $34.7 \%(17 / 49)$ of tumors, smaller in $40.8 \%(20 / 49)$ of tumors, and identical in $24.5 \%$ (12/49) of tumors. Overall, measurements taken from pathology specimens were smaller than those obtained from radiographs $(r=0.92$, $P<0.001$; see Fig. 4), with pathology specimen measurements reduced by $8 \%$ relative to those from radiographs.

The radiograph-slide contraction coefficient was $82.9 \%$ $(0.92 \times 0.90)$, and a distance of $5 \mathrm{~mm}$ was required to include $98 \%$ of microextensions. By extrapolation, the equivalent distance on radiographic specimens had to be $6.1 \mathrm{~mm}(5.0 / 0.829)$. For a score of $\leq 1$, an expansion distance of $2.41 \mathrm{~mm}(2.0 / 0.829)$ on radiographic images can be accepted, which accounts for $\geq 95 \%$ probability of microextension. For a score of $\geq 2$, an expansion distance

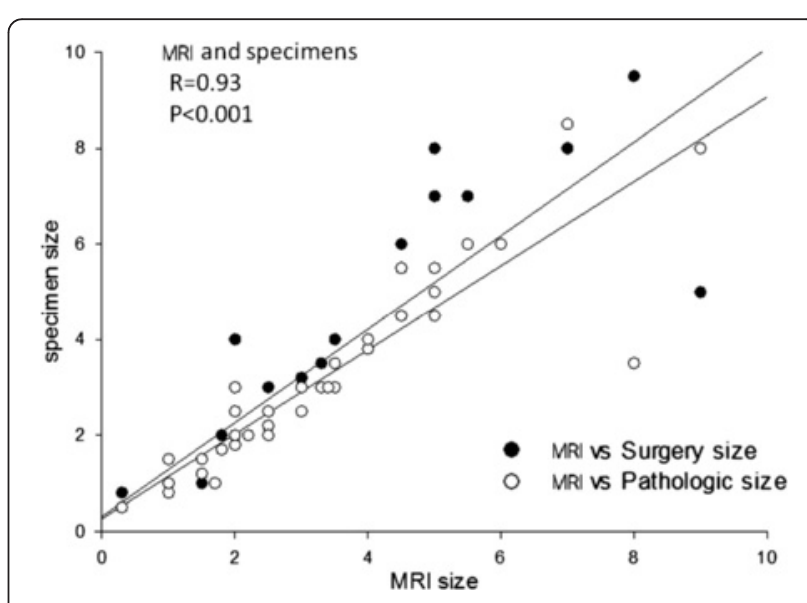

Fig. 4 Correlation between tumor sizes measured using magnetic resonance images and pathology specimen slides 
of $6.1 \mathrm{~mm}$ on radiographic images must be selected. A GTV-to-CTV expansion of $8.5 \mathrm{~mm}$ in radiographic images of LMCRC is required to encompass the gross tumor and all subclinical microscopic disease with $100 \%$ accuracy.

\section{Discussion}

The liver is the target of many metastatic cancers, particularly those of colorectal cancer. Major advances in the diagnosis and treatment of metastatic liver cancers have occurred during the last two decades. Surgery is still considered the gold-standard therapy for patients diagnosed with LMCRC because it confers the best chance of long-term survival. However, only $25 \%$ of patients with liver metastases are candidates for curative resection [2]. The majority are not eligible for surgery due to extensive disease, multiplicity of tumor, concomitant major systemic disease, or poor hepatic reserve. During the past 20 years, SBRT has evolved as another local treatment option for primary and metastatic liver tumors. Local control rates have been improved by dose escalation protocols, whereas acceptable levels of toxicity have been maintained $[6,7,12,13]$.

To further optimize SBRT treatment, the definition of the target volume needs to be improved. Determining the degree of GTV expansion to include the CTV poses a considerable challenge to radiologists. To precisely define the limits of the target volume, the correlation between macroscopic tumor dimensions that are visible in radiological images and those that are visible in pathology specimens should be evaluated. To our knowledge, reports in the literature on this topic are scarce $[14,15]$. The current study was designed as a prospective pilot study to establish all the necessary procedures to obtain a good clinicopathologic correlation between the methodologies and reproducible microextension measurements.

We found that expansions of 5.0 or $6.7 \mathrm{~mm}$ in microscopy specimens were required to encompass the gross tumor and any microscopic disease with $95 \%$ or $99 \%$ accuracy, respectively. A more important issue was to measure the tissue contraction coefficient that occurs after formalin fixation of specimens and syneresis in the automatic tissue hydroextractor. Our analysis of 66 liver carcinoma pathology specimens on slides revealed that the prepared specimen sizes were $91.4 \%$ of the original specimens. The radiograph-slide contraction coefficient was $82.9 \%(0.92 \times 0.90)$. These calculations indicate that an expansion of at least $6.1 \mathrm{~mm}$ on radiographic images should be considered during radiological treatment of LMCRC.

The microextension frequency observed in the present study is in agreement with other published reports. Prospective and retrospective surgical reports describe the presence of microextensions with a large variation
$(2-58 \%)$ in occurrence $[16,17]$. Our results were in agreement with those of Gandhi et al., who reported measurable microscopic disease (mean, $1.25 \mathrm{~mm}$ ) in 7 of $24(29.2 \%)$ tumors analyzed [18]. However, the depths of microextension infiltration $(0.15-38.00 \mathrm{~mm})$ described in the literature are not consistent with our results, which ranged from $1-7 \mathrm{~mm}$. One possible reason for the discrepancy could be that the criteria for patient selection differed in the two studies. Furthermore, it is impossible to entirely exclude whether some of our tumor nests (observed in a two-dimensional microscopic field) were not in reality attached to the primary tumor at another level, because some of the colorectal metastases had very irregular borders. We tried to correct for this weakness as much as possible by thoroughly inspecting the slices located just above and beneath the one in which we observed the microextension. In general, all the metastases included in the present study showed rather irregular shapes, and this made it difficult to establish a relationship between the irregular tumor shape and more frequent or deeper microextensions.

We found that microextension was related to the original primary tumor site, CEA levels, and multiple lesions. Reports on prognosis in colon and rectal cancers are limited. In our study, the median microextension levels in liver were significantly different depending on whether the origin of metastasis was from the colon or rectum. A possible reason for this may be the differences in their anatomical locations, which could account for different pathways in lymph node metastases and specific biological behaviors. However, further study is needed to understand the phenomenon.

There are some reports that serum CEA level is an independent risk factor for LMCRC. Patients with elevated CEA levels are more likely to develop recurrent disease and to have poorer overall survival than patients with normal CEA levels $[19,20]$. Our results are in agreement with this assessment. The presence of multiple lesions is another factor that correlates with microextension, although the $P$ value was only 0.045 . The presence of multiple lesions suggests that the cancer cells are more highly prone to invasion.

It is unclear whether pretreatment with chemotherapy might influence metastatic microextensions. A limitation in our study is that the impact of tumor regression may not be the same after treatment with 5-fluorouracil (5-FU) or tritherapy with or without targeted agents such as the anti-VEGF monoclonal antibody bevacizumab. In our study, analysis of patients with or without chemotherapy did not identify any significant differences in microextension $(P=0.906)$. We used a specimen-slide contraction coefficient that was derived only from primary liver carcinoma specimens. We do not currently know if the 
LMCRC specimens would have the same coefficient. Additional research is needed to answer this question. Only 49 patients had a preoperative MRI scan in our study, which may lead to biases in the results. Most of the 129 patients included in the study had a preoperative ultrasonic sound examination. Only patients with an obscure mass or a small tumor were referred for additional examination using CT or MRI. This referral was dependent on the surgeon's decision.

Analysis of the cumulative distribution of microinvasion in LMCRT patients with different tumor characteristics (Fig. 3) revealed that the CEA level was the best predictor of microextension. However, the pathologic stage cannot be accurately determined without surgery. Therefore, we believe that the scoring system developed in this paper, which is based on factors that can be accurately determined preoperatively (i.e., CEA level, original primary tumor site, and number of liver metastases), will hold greater promise for prognosis.

The average tumor size in this patient cohort was $2.99 \mathrm{~cm}$. Considering that a tumor is approximated as a sphere, a reduction in the GTV-to-CTV expansion from $6.1 \mathrm{~mm}$ (score $\geq 2$ ) to $2.41 \mathrm{~mm}$ (score $\leq 1)$ would reduce the CTV diameter from 4.21 to $3.47 \mathrm{~cm}$. Accordingly, the CTV volumes would be reduced from 39.05 to $21.87 \mathrm{~cm}^{3}$ (i.e., a $50 \%$ reduction). This suggests that the delineation of CTV could vary depending on differences in patientspecific clinical characteristics. Another important point is that the average tumor size in our cohort was very small $(2.99 \mathrm{~cm})$. Further studies based on our analyses will facilitate potential clinical applications of this promising methodology for conformal radiotherapy of LMCRT patients.

\section{Conclusions}

This study showed that an expansion of $2.41 \mathrm{~mm}$ on radiographic images with a score of $\leq 1$ can be accepted; however, to account for $98 \%$ probability of microextension, an expansion of $6.1 \mathrm{~mm}$ on radiographic images with a score of $\geq 2$ must be selected. A GTV-to-CTV expansion of $8.5 \mathrm{~mm}$ in radiographs of LMCRT patients is required to encompass the gross tumor and all microscopic disease with $100 \%$ accuracy. Microextension distance is correlated with original primary tumor site, serum CEA levels, and multiple metastatic lesions in liver. Our multifactor scoring system, which takes CEA level into consideration, was a good predictor of microextension in our dataset.

\footnotetext{
Abbreviations

LMCRC: Liver metastases from colorectal cancer; GTV: Gross tumor volume; CTV: Clinical tumor volume; CEA: Carcinoembryonic antigen; CA199: Carbohydrate antigen 199; SBRT: Stereotactic body radiation therapy; PBS: Phosphate-buffered saline; MRI: Magnetic resonance imaging; CT: Computed tomography; 5-FU: 5-fluorouracil.
}

\section{Competing interests}

The authors declare that they have no competing interests.

\section{Authors' contributions}

YQ: implemented a scoring system for determining GTV to CTV expansion, was involved in the collection of data, the design of study drafting of the manuscript, and the critical revision of the manuscript for scientific content. ZCZ: conceived of the study, and participated in its design and coordination and helped to draft the manuscript. YJ: assessed all specimens to identify microextension and interpretation of data. YPX: assessed all specimens to identify microextension and the critical revision of the manuscript for scientific content. All authors read and approved the final manuscript.

\section{Acknowledgement}

The authors would like to thank colleagues of pathological department for their technical supports.

\section{Author details}

'Department of Radiation Oncology, Zhongshan Hospital, Fudan University, 136 Yi Xue Yuan Road, Shanghai 200032, China. ${ }^{2}$ Department of Pathology, Zhongshan Hospital, Fudan University, Shanghai, China.

Received: 11 October 2014 Accepted: 25 May 2015

Published online: 04 June 2015

\section{References}

1. Poston GJ. Surgical strategies for colorectal liver meatstases. Surg Oncol. 2004;13:125-36.

2. Kemeny N. Management of liver metastases from colorectal cancer. J Oncol. 2006;20:1 161-79.

3. van der Pool AE, Méndez Romero A, Wunderink W, Heijmen BJ, Levendag $P C$, Verhoef $C$, et al. Stereotactic body radiation therapy for colorectal liver metastases. Br J Surg. 2010;97:377.

4. Dawood O, Mahadevan A, Goodman KA. Stereotactic body radiation therapy for liver metastases. Eur J Cancer. 2009;45(17):2947-59.

5. Rusthoven KE, Kavanagh BD, Cardenes H, Stieber WW, Burri SH, Feigenberg $\mathrm{SJ}$, et al. Multi-institutional phase I/II trial of stereotactic body radiation therapy for liver metastases. J Clin Oncol. 2009;27:1572-8.

6. Chang DT, Swaminath A, Kozak M, Weintraub J, Koong AC, Kim J, et al. Stereotactic body radiotherapy for colorectal liver metastases: a pooled analysis. Cancer. 2011;117:4060-9.

7. Okano K, Yamamoto J, Kosuge T, Yamamoto S, Sakamoto M, Nakanishi Y, et al. Fibrous pseudocapsule of metastatic liver tumors from colorectal carcinoma: clinicopathologic study of 152 first resection cases. Cancer. 2000;89:267-75.

8. Baumert BG, Rutten I, Hing-Oberije C, Twijnstra A, Dirx MJ, DebougnouxHuppertz RM, et al. A pathologybased substrate for target definition in radiosurgery of brain metastases. Int J Radiat Oncol Biol Phys. 2006;66:187-94.

9. Balci NC, Befeler AS, Leiva P, Pilgram TK, Havlioglu N. Imaging of liver disease: comparison between quadruple-phase multidetector computed tomography and magnetic resonance imaging. J Gastroenterol Hepatol. 2008;23:1520-7.

10. Cantwell CP, Setty BN, Holalkere N, Sahani DV, Fischman AJ, Blake MA. Acomparison of low radiation dose non-enhanced PET/CT, contrast-enhanced PET/CT, and liver MRI. J Comput Assist Tomogr. 2008;32:738-44.

11. Bi AH, Zeng ZC, Ji Y, Zeng HY, Xu C, Tang ZY, et al. Impact factors for microinvasion in intrahepatic cholangiocarcinoma: a possible system for defining clinical target volume. Int J Radiat Oncol Biol Phys. 2010;78(5):1427-36.

12. Lee MT, Kim JJ, Dinniwell R, Brierley J, Lockwood G, Wong R, et al. Phase I study of individualized stereotactic body radiotherapy of liver metastases. J Clin Oncol. 2009;27:1585-91.

13. Schefter TE, Kavanagh BD, Timmerman RD, Cardenes HR, Baron A, Gaspar LE. A phase I trial of stereotactic body radiation therapy (SBRT) for liver metastases. Int J Radiat Oncol Biol Phys. 2005;62:1371-8.

14. Dawson LA, Brock KK, Moulton C, Moseley J, Ahmed S, Guindi M, et al. Comparison of liver metastases volumes on CT, MR and FDG PET imaging to pathological resection using deformable image registration [abstract]. Int J Radiat Oncol Biol Phys. 2007;69(Suppl1):68.

15. Semelka RC, Hussain SM, Marcos HB, Woosley JT. Perilesional enhancement of hepatic metastases: correlation between MR imaging and histopathologic findings: Initial observations. Radiology. 2000;215:89-94. 
16. Ambiru S, Miyazaki M, Isono T, Ito H, Nakagawa K, Shimizu H, et al. Hepatic resection for colorectal metastases: analysis of prognostic factors. Dis Colon Rectum. 1999;42:632-9.

17. Wakai T, Shirai Y, Sakata J, Valera VA, Korita PV, Akazawa K, et al. Appraisal of $1 \mathrm{~cm}$ hepatectomy margins for intrahepatic micrometastases in patients with colorectal carcinoma liver metastasis. Ann Surg Oncol. 2008;15:2472-81.

18. Ghandi D, Mitchell KA, Miick R, Chang BW. Clinicopathologic analysis of gross tumor size and microscopic extension in liver metastases from colorectal cancer (CRC): implications for stereotactic body radiotherapy (SBRT) [abstract]. Int J Radiat Oncol Biol Phys. 2009;75(Suppl1):29.

19. Andicoechea A, Vizoso F, Alexandre E, Cuesta E, Cruz Díez M, Miera L, et al. Preoperative carbohydrate antigen 195 (CA195) and CEA serum levels as prognostic factors in patients with colorectal cancer. Int J Biol Markers. 1998;13:158-64.

20. Rees M, Tekkis PP, Welsh FK, O'Rourke T, John TG. Evaluation of long-term survival after hepatic resection for metastatic colorectal cancer:a multifactorial model of 929 patients. Ann Surg. 2008;247(1):125-35

\section{Submit your next manuscript to BioMed Central and take full advantage of:}

- Convenient online submission

- Thorough peer review

- No space constraints or color figure charges

- Immediate publication on acceptance

- Inclusion in PubMed, CAS, Scopus and Google Scholar

- Research which is freely available for redistribution 